\title{
Disjunction of LOTOS specifications
}

\author{
M.W.A. Steen, H. Bowman, J. Derrick and E.A. Boiten \\ University of Kent at Canterbury \\ Computing Lab., The University, Canterbury, Kent CT2 7NF, UK. \\ Email:mwas@ukc.ac.uk
}

\begin{abstract}
LOTOS is a formal specification language, designed for the precise description of open distributed systems and protocols. The definition of, so called, implementation relations has made it possible also to use LOTOS as a specification technique for the design of such systems. These LOTOS based specification techniques usually (ab)use non-determinism to achieve implementation freedom. Unfortunately, this is unsatisfactory when specifying non-deterministic processes. We, therefore, propose to extend LOTOS with a disjunction operator in order to achieve more implementation freedom while maintaining the possibility to describe non-deterministic processes. In contrast with similar proposals we maintain the operational semantics.
\end{abstract}

\section{Keywords}

LOTOS, process algebra, specification, disjunction, operational semantics

\section{INTRODUCTION}

In this paper we investigate the extension of the formal specification language LOTOS with a disjunction operator. Such a specification construct could play a role in achieving a more expressive specification technique. As in logic, disjunction can be used to specify a choice between implementation options. If $p_{1}$ is an implementation of $s_{1}$, and $p_{2}$ is an implementation of $s_{2}$, then the specification $s_{1} \bigvee s_{2}$ can be implemented by either $p_{1}$ or $p_{2}$. Thus, disjunction in specifications leads to greater implementation freedom. This is useful both in the specification of standards, which often describe a number of implementation classes, and in the development of distributed systems, where we do not want to tie the hands of the implementors in the initial specification.

\subsection{Interpreting LOTOS specifications}

LoTOS is a process algebraic language influenced by the earlier process calculi CCS [Mi189] and CSP [Hoa85]. For example, it has inherited the powerful idea of multi-way synchronisation, enabling constraint-oriented specification, from CSP. On the other hand, the language has been given an operational semantics much in the style of CCS. 


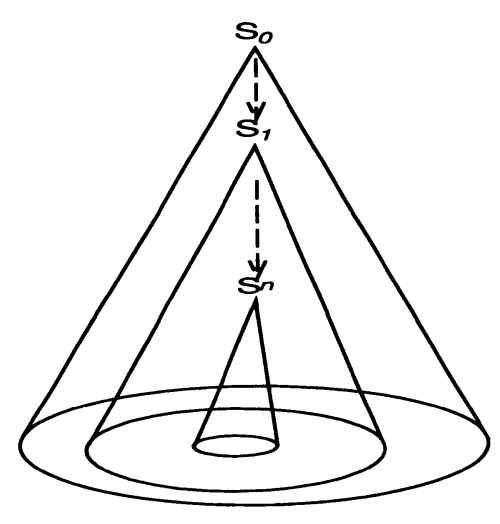

Figure 1 Design process

The operational semantics associates a labelled transition system (LTS) with each process description. The usual interpretation of a LOTOS "specification" is the set of processes that are indistinguishable (w.r.t. some notion of equivalence) from the LTS associated with it. However, this view requires that the observational behaviour of implementations is completely determined already by their initial specifications.

An alternative, more relaxed, view is, that implementations are related to specifications by a, so called, implementation relation*. Implementation relations are usually not equivalences and, therefore, allow the behaviour of implementations to be somehow more determined than the behaviour described by their specifications. Moreover, they induce a refinement ordering between specifications, which enables an incremental design process as depicted in figure 1 . An initial abstract specification, allowing many possible implementations, goes through a series of consecutive refinement steps, each restricting the implementation space, until a final implementation is reached.

\subsection{The problem}

Several researchers have investigated the use of implementation relations with LOTOS to obtain a specification technique for concurrent processes (see section 3). Most of these approaches are inspired by CSP's failures/divergences semantics, or have been derived from testing theory. Non-determinism is usually (ab)used to achieve implementation freedom. We argue that this is not always satisfactory. In particular, we show that it becomes impossible to specify inherently non-deterministic processes adequately, and the wide-spread use of internal actions as an abstraction mechanism can lead to counter-intuitive implementations.

* Implementation relations are sometimes also referred to as conformance relations or satisfaction relations. 


\section{LOTOS: SYNTAX AND SEMANTICS}

In order not to clutter the presentation of our main ideas, we will only consider a small subset of the operators that LOTOS offers for the structuring of process descriptions. The subset we use is inductively defined by the following grammar:

$P::=$ stop $|a ; P| i ; P \mid P[P|P|[G]|P| X$

Here we assume that a set of action labels $\mathcal{L}$ is given. Then, $a \in \mathcal{L} ; i$ is the unobservable, or internal, action; $G \subseteq \mathcal{L}$; and $X$ is a process name. We will assume that a definition exists for each process name used. Process definitions are written $X:=P$, where $P$ is a behaviour expression that can again contain process names, including possibly $X$ itself, thus making the definition recursive. The set of all processes is denoted by $\mathcal{P}$, elements of $\mathcal{L}$ by $a, b, c \ldots$, and elements of $\mathcal{L} \cup\{i\}$ by $\mu$.

The operational semantics for LOTOS associates a labelled transition system with each behaviour description through the axioms and inference rules given in table 1.

Table 1 Inference rules

\begin{tabular}{lll}
\hline & $\vdash$ & $a ; P \stackrel{a}{\longrightarrow} P$ \\
& $\vdash$ & $i ; P \stackrel{i}{\longrightarrow} P$ \\
$P \stackrel{\mu}{\longrightarrow} P^{\prime}$ & $\vdash$ & $P\left[Q \stackrel{\mu}{\longrightarrow} P^{\prime}\right.$ \\
$Q \stackrel{\mu}{\longrightarrow} Q^{\prime}$ & $\vdash$ & $P\left[Q \stackrel{\mu}{\longrightarrow} Q^{\prime}\right.$ \\
$P \stackrel{\mu}{\longrightarrow} P^{\prime}, \mu \notin G$ & $\vdash$ & $P|[G]| Q \stackrel{\mu}{\longrightarrow} P^{\prime}|[G]| Q$ \\
$Q \stackrel{\mu}{\longrightarrow} Q^{\prime}, \mu \notin G$ & $\vdash$ & $P|[G]| Q \stackrel{\mu}{\longrightarrow} P|| G] \mid Q^{\prime}$ \\
$P \stackrel{a}{\longrightarrow} P^{\prime}, Q \stackrel{a}{\longrightarrow} Q^{\prime}, a \in G$ & $\vdash$ & $P|[G]| Q \stackrel{a}{\longrightarrow} P^{\prime} \mid[G] Q^{\prime}$ \\
$P \stackrel{\mu}{\longrightarrow} P^{\prime}, X:=P$ & $\vdash$ & $X \stackrel{\mu}{\longrightarrow} P^{\prime}$ \\
\hline
\end{tabular}

The LTS for a process $p$ is $\left\langle D_{p}, L_{p}, T_{p}, p\right\rangle$. Here $T_{p}$ is the smallest set of transitions that can be inferred from $p$ under the given inference rules; $D_{p}$ is the set of processes derivable from $p$ under the transitions in $T_{p} ; L_{p}=\left\{a \mid\left(s, a, s^{\prime}\right) \in T_{p}\right\}$, the set of action labels.

\subsection{Further notation}

For the rest of the paper we need some more derived notation. Let $\mathcal{L}^{*}$ denote strings over $\mathcal{L}$. The constant $\epsilon \in \mathcal{L}^{*}$ denotes the empty string, and the variables $\sigma, \sigma_{i}$ are used to range over $\mathcal{L}^{*}$. Elements of $\mathcal{L}^{*}$ are also called traces. In table 2 the notion of transition is generalised to traces. We further define $\operatorname{Tr}(p)$, the set of traces of $p$, and $\operatorname{Ref}(p, \sigma)$, the sets of actions refused by $p$ after the trace $\sigma$ :

$\operatorname{Tr}(p)=\left\{\sigma \in L^{*} \mid p \stackrel{\sigma}{\Longrightarrow}\right\}$

$\operatorname{Ref}(p, \sigma)=\left\{X \subseteq L \mid \exists p^{\prime}: p \stackrel{\sigma}{\Longrightarrow} p^{\prime}\right.$ and $\left.\forall a \in X: p^{\prime} \stackrel{a}{\Rightarrow}\right\}$. 
Table 2 Derived transition denotations

\begin{tabular}{cl}
\hline Notation & Meaning \\
\hline$p \stackrel{\mu}{\longrightarrow}$ & $\exists p^{\prime}: p \stackrel{\mu}{\longrightarrow} p^{\prime}$ \\
$p \stackrel{\mu}{\hookrightarrow}$ & $\nexists p^{\prime}: p \stackrel{\mu}{\longrightarrow} p^{\prime}$ \\
$\stackrel{\epsilon}{\Longrightarrow}$ & reflexive and transitive closure of $\stackrel{i}{\longrightarrow}$ \\
$p \stackrel{a \sigma}{\longrightarrow} p^{\prime}$ & $\exists q, q^{\prime}: p \stackrel{\epsilon}{\Longrightarrow} q \stackrel{a}{\longrightarrow} q^{\prime} \stackrel{\sigma}{\longrightarrow} p^{\prime}$ \\
$p \stackrel{\sigma}{\Longrightarrow}$ & $\exists p^{\prime}: p \stackrel{\sigma}{\longrightarrow} p^{\prime}$ \\
$p \stackrel{\sigma}{\Rightarrow}$ & $\nexists p^{\prime}: p \stackrel{\sigma}{\Longrightarrow} p^{\prime}$ \\
\hline
\end{tabular}

\subsection{Equivalence}

Often transition systems are considered to be too discriminating in the sense that processes that are intuitively considered to be equivalent may have different representations. The processes $a ; b ;$ stop and $a ;(b ;$ stop $[b ;$ stop) $[a ; b ;$ stop, for example, have different transition systems, but can both only perform the sequence of actions $a b$ and then deadlock. For this reason several abstracting equivalences have been defined over the LTS model. In this paper, we consider only the strongest of the behavioural equivalences: strong bisimulation equivalence. Processes are equivalent iff they can simulate each other. This is indeed the case for the two processes above.

\section{Definition 1 (bisimulation equivalence)}

Bisimulation equivalence, $\sim \subseteq \mathcal{P} \times \mathcal{P}$, is the largest relation such that, $p \sim q$ implies

(i) Whenever $p \stackrel{\mu}{\longrightarrow} p^{\prime}$ then, for some $q^{\prime}, q \stackrel{\mu}{\longrightarrow} q^{\prime}$ and $p^{\prime} \sim q^{\prime}$; and

(ii) Whenever $q \stackrel{\mu}{\longrightarrow} q^{\prime}$ then, for some $p^{\prime}, p \stackrel{\mu}{\longrightarrow} p^{\prime}$ and $p^{\prime} \sim q^{\prime}$.

The choice of equivalence is fairly arbitrary. We could just as well have chosen weak bisimulation equivalence or testing equivalence. We are, however, interested in creating a specification technique that is as expressive as possible. Since bisimulation equivalence is the strongest behavioural equivalence on processes, and by defining satisfaction (see section 4 ) as an extension of it, we achieve precisely this.

\section{LOTOS AS A SPECIFICATION TECHNIQUE}

The "meaning" of a specification, i.e. the set of implementations that it describes, depends on the chosen satisfaction relation. Following [Lar90a] and [Led92], we define a specification technique to be a pair $\langle\Sigma$, sat $\rangle$, where $\Sigma$ is the set of all specifications, and sat is some satisfaction relation. Using the notion of bisimulation from the previous section, we could instantiate sat with $\sim$. However, as argued in the introduction, this would leave very little room for manoeuvring during the implementation phase, because the behaviour of implementations would have to be equivalent to the behaviour of their specifications. 
Several asymmetric instantiations for sat have been investigated for LOTOS [BSS87, Led91]. These, so called, implementation relations were either derived from CSP's denotational semantics [Hoa85], or from testing theory [NH84].

One of the simplest implementation relations, is the trace preorder. It only verifies that the implementation cannot perform sequences of observable actions (traces) that are not allowed by the specification.

Definition 2 (trace preorder) Let $p, s \in \mathcal{P}$. $p \leq_{t r} s$ iff $\operatorname{Tr}(p) \subseteq \operatorname{Tr}(s)$.

Example 1 Let $s:=a ; b ;$ stop $\llbracket a ; c ;$ stop, then $p_{1}:=a ; b ;$ stop, $p_{2}:=a ; c ;$ stop and $p_{3}:=a ;(b ;$ stop $\square c ;$ stop $)$ are all implementations of $s$ according to $\leq_{t r}$. But, also stop and $a$; stop are correct, since $\leq_{t r}$ does not require any behaviour to be implemented.

The trace preorder is a very weak implementation relation. We cannot use it to specify that anything must happen. Another notion of validity is, that for each trace of the specification, the implementation can only refuse whatever the specification refuses after that trace. This is captured by the conf-relation, which was derived from testing theory. Here we give an intensional definition in terms of traces and refusals.

Definition 3 (conf) Let $p, s \in \mathcal{P}$. $p$ conf $s$ iff $\forall \sigma \in \operatorname{Tr}(s): \operatorname{Ref}(p, \sigma) \subseteq \operatorname{Ref}(s, \sigma)$.

Example 2 For the specifications and processes given in example $1, p_{1}, p_{2}$ and $p_{3}$ are all correct implementations of $s$ according to conf. However, stop and $a$; stop are not, because s requires either b or $c$ to happen after $a$.

The relation red (sometimes referred to as testing preorder, or failure preorder), which is the intersection of $\leq_{t r}$ and conf, gives rise to a specification technique with which we can specify both that certain actions must happen and that certain traces are not allowed. This seems to give a suitable specification technique for concurrent processes.

Example 3 Suppose we want to specify a class of drinks machines. All machines should initially accept a coin. After that, the implementations should give the user either coffee or tea, or a choice between both. With $\langle\mathcal{P}$, red $\rangle$ we can capture this class of behaviours with the following specification:

$$
\mathrm{s}:=\text { coin; (i; coffee; stop [] i; tea; stop) }
$$

In the example above, note that $s$ also allows the implementation that non-deterministically offers either coffee or tea, after accepting a coin. Since the non-determinism is solely used for achieving implementation freedom in the specification, we could require that implementations are fully deterministic. In that case we have a specification technique that is suitable for specifying deterministic processes.

Unfortunately, non-determinism is not only used to specify implementation free- 
dom. There are some inherently non-deterministic systems, such as gambling machines. More importantly, non-determinism is needed to model non-deterministic aspects of the environment that we do not control. Examples are lossy, or erroneous communication media. In addition, the LOTOS internal action is sometimes used to model certain implementation details that cannot be modelled in LOTOS. In the example below, we show how reduction of non-determinism can lead to intuitively incorrect implementations in these cases.

Example 4 In the following specification of a transmission protocol, the internal action is used to abstract from the occurrence of a timeout, which is currently not explicitly expressible in LOTOS.

$$
\mathrm{TP}_{\text {spec }}:=\text { send; (receive_ack; stop [] i (* timeout *); error; stop ) }
$$

This protocol sends a packet and then waits for an acknowledgement. If the acknowledgement is not received within a certain time, the protocol gives an error signal.

According to red, this specification can be implemented by a process that gives an error straight away, which is counter-intuitive.

$$
\mathrm{TP}_{\text {error }}:=\text { send; error; stop }
$$

Many more implementation relations exist, but most of them are also based on the assumption that implementations may be more deterministic than specifications. Implementation relations that require implementations to be as deterministic as their specifications are usually equivalence relations, which we have rejected for other reasons.

The solution we pursue in the next section separates the use of non-determinism to achieve implementation freedom from its other uses. A new specification construct is introduced for the specification of implementation options. An implementation is then a (possibly non-deterministic) specification in which all the implementation options have been resolved.

\section{DISJUNCTION}

In this section, we propose to extend LOTOS with a specification construct for explicitly specifying alternative implementation options. The construct we envisage has similarities to CSP's internal choice, but is closer to logical disjunction. In CSP the specification $P \sqcap Q$ could be implemented by $P \square Q$, but in logic, either $P$ or $Q$ would satisfy $P \vee Q$ (the choice is exclusive). The operator will be called disjunction, and denoted by $V$, because its properties are very much like those of logical disjunction. In the following $\mathcal{S}$ denotes the set of all specifications satisfying this extended syntax.

Disjunction is an operation on specifications that can be used to compose requirements that do not have to be satisfied simultaneously. In order to satisfy the specification $s \bigvee t$ it is enough to implement either $s$ or $t$. Disjunction is a specification construct. Disjunctions cannot occur in implementations. Therefore disjunctions should 
Table 3 Inference rules for unlabelled transitions

\begin{tabular}{|c|c|c|}
\hline$s \longmapsto s^{\prime}$ & & $s \rrbracket t \longmapsto s^{\prime} \rrbracket t$ \\
\hline$t \longmapsto t^{\prime}$ & $\vdash$ & $s \rrbracket t \longmapsto s \rrbracket t^{\prime}$ \\
\hline$s \longmapsto s^{\prime}$ & $\vdash$ & $s|[G]| t \longmapsto s^{\prime}|[G]| t$ \\
\hline$t \mapsto t^{\prime}$ & $\vdash$ & $s|[G]| t \longmapsto s|[G]| t^{\prime}$ \\
\hline$s \longmapsto s^{\prime}, x:=s$ & $\vdash$ & $x \longmapsto s^{\prime}$ \\
\hline
\end{tabular}

gradually be eliminated from the specification during consecutive refinement steps. Refinement should not reduce non-determinism though.

In order to define the semantics for disjunction operationally, we augment labelled transition systems with a new, unlabelled, transition: $\rightarrow$. These unlabelled transitions can only be introduced by the disjunction operator through the following axioms:

$$
\frac{-}{s \bigvee t \longmapsto s} \quad \frac{-}{s \bigvee t \longmapsto t}
$$

i.e., a disjunction can be resolved through an unlabelled transition. The operational semantics of a specification is now given by an augmented labelled transition system.

\section{Definition 4 (Augmented Labelled Transition System)}

An augmented labelled transition system (ALTS) is a structure $\left\langle S, L, \rightarrow, \mapsto, s_{0}\right\rangle$, with $S$ a set of states, $L$ a set of action labels, $\rightarrow \subseteq S \times L \cup\{i\} \times S$ a set of labelled transitions, $\longrightarrow \subseteq S \times S$ a set of unlabelled transitions, and $s_{0} \in S$ the initial state.

The ALTS for a specification is determined in the usual fashion by the axioms for disjunction given above, and a set of inference rules. The inference rules that determine the normal transition relation, $\rightarrow$, are the same as the normal transition rules for LOTos given in table 1 . The rules for unlabelled transitions are given in table 3 . Note that unlabelled transitions are just passed through by all binary operators and recursion. The reason for this is that we do not want a choice, for example, to be resolved by the presence of a disjunction in one of its arguments.

Example 5 Below we have depicted the transition systems for the specifications $S_{1}:=a ;$ stop $\bigvee b ;$ stop and $S_{2}:=(a ;$ stop $\square b ;$ stop $) \bigvee(a ;$ stop $\square c ;$ stop $)$
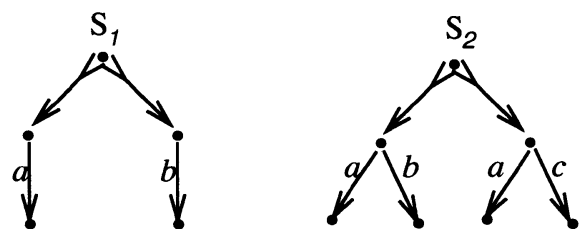

In case of nested disjunctions (see example 6) we will usually not be interested in the disjuncts that are again disjunctions themselves. Our interest will be in the "real" 
disjuncts, i.e., those states that can be reached through a sequence of unlabelled transitions, but which have no outgoing unlabelled transitions themselves. In the remainder of this paper, we therefore use a derived disjunction relation, defined below.

Example 6 Depicted below is the transition system for $a$; $\operatorname{stop} \bigvee(b ;$ stop $\bigvee c$; stop).

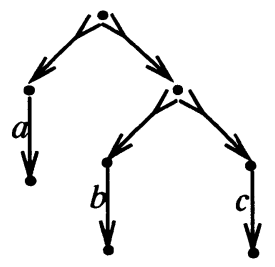

\section{Definition 5 (derived disjunction relations)}

1. For a specification $s$, we define the following predicates:

$$
\begin{array}{lll}
s \mapsto \quad \text { iff } & \exists s^{\prime}: s \mapsto s^{\prime} & \text { (s is a disjunction) } \\
s \not \nrightarrow \quad \text { iff } \quad \nexists s^{\prime}: s \mapsto s^{\prime} & \text { (s is not a disjunction) }
\end{array}
$$

2. For specifications $s$ and $t$, we define the following relations:

$$
\begin{array}{lll}
s \mapsto^{*} t \quad \text { iff } \quad t=s \vee \exists s^{\prime}: s \mapsto s^{\prime} \wedge s^{\prime} \mapsto^{*} t & \\
& \text { (i.e., the reflexive and transitive closure of } \mapsto) \\
s \mapsto^{*} t \quad \text { iff } \quad & s \mapsto^{*} t \wedge t \not t
\end{array}
$$

The following lemma gives two useful properties for the $\nrightarrow^{*}$-relation.

\section{Lemma 1}

1. $s \not \longleftrightarrow \Longleftrightarrow s \leftrightarrow^{*} s$;

2. $s \bigvee t \leftrightarrow^{*} x \Longleftrightarrow s \leftrightarrow^{*} x \vee t \leftrightarrow^{*} x$.

Proof.

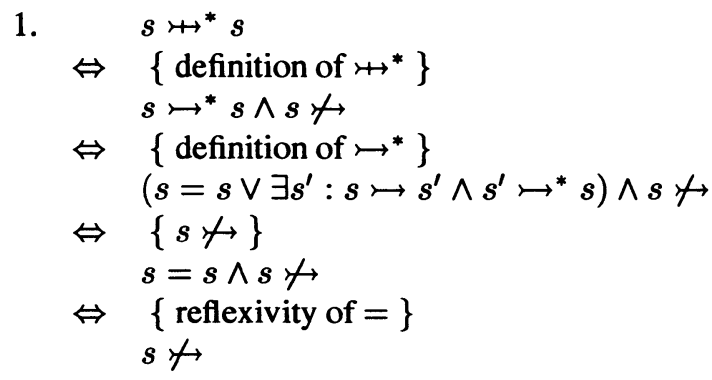




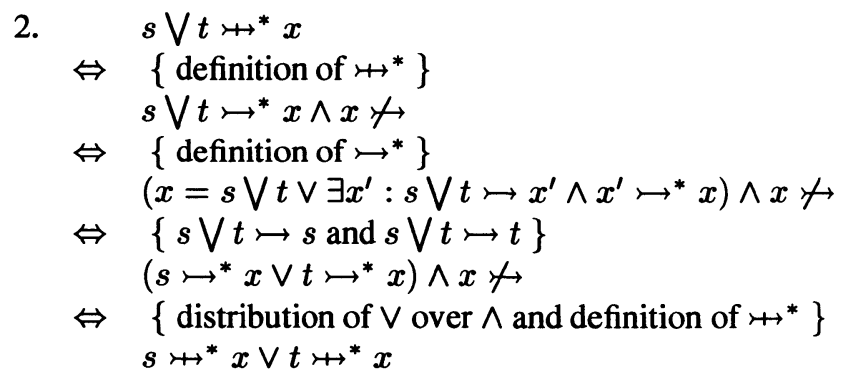

So far, there is nothing much new. The unlabelled transitions could just as well have been internal actions. The relation $\leftrightarrow^{*}$ would then correspond to the relation given by $\{(s, t) \mid s \stackrel{\epsilon}{\Rightarrow} t \wedge t \stackrel{i}{\not t}\}$. However, by introducing a different transition, we separate the specification of alternative implementation options from the use of internal actions and non-determinism. Note that rather than introducing an extra transition relation, we could have introduced another special action label like the $i$ for internal actions.

In the following two sections, we define satisfaction and refinement as extensions of bisimulation equivalence. This is where we deviate from the usual approaches based on refusals.

\subsection{Satisfaction}

From here on we distinguish between processes, or implementations, which have no disjunctions, and specifications, which may have disjunctions. Processes are in the set $\mathcal{P}$, and specifications are drawn from the set $\mathcal{S}$.

A process intuitively satisfies a specification in case it is equivalent to one of its disjuncts. This intuition is reflected by the formal definition of satisfaction below. Since each disjunct can again have further disjuncts, the definition is inductive. Observe that we have used a "strong" interpretation. There is, however, no reason why this schema could not be applied to weaker interpretations of equivalence, provided they can be characterised inductively.

\section{Definition 6 (Satisfaction)}

Satisfaction, $\models \subseteq \mathcal{P} \times \mathcal{S}$, is the largest relation such that, $p \models s$ implies $\exists s^{\prime}: s \uplus^{*} s^{\prime}$ and, for each $\mu \in L \cup\{i\}$ the following two conditions hold:

$\left(\vDash_{1}\right) \quad$ Whenever $p \stackrel{\mu}{\longrightarrow} p^{\prime}$, then $s^{\prime} \stackrel{\mu}{\longrightarrow} s^{\prime \prime}$ for some $s^{\prime \prime}$ with $p^{\prime} \vDash s^{\prime \prime}$; and

$\left(\vDash_{2}\right) \quad$ Whenever $s^{\prime} \stackrel{\mu}{\longrightarrow} s^{\prime \prime}$, then $p \stackrel{\mu}{\longrightarrow} p^{\prime}$ for some $p^{\prime}$ with $p^{\prime} \vDash s^{\prime \prime}$.

Now, we can instantiate $s a t$ with $\models$ to obtain a powerful specification technique for both deterministic and non-deterministic processes. 
Example 7 Going back to the drinks machine specification of example 3, we can now specify the class of drinks machines that serve either coffee or tea as follows:

$$
\mathrm{S} 1:=\text { coin; (coffee; stop } \bigvee \text { tea; stop) }
$$

Possible implementations, according to $\models$, are: coin; coffee; stop and coin; tea; stop. If we also want to allow the implementation that offers a choice between coffee and tea, after a coin has been accepted, then we should add this as a disjunct to the specification:

$$
\text { S2 := coin; (coffee; stop } \bigvee \text { tea; stop } \bigvee \text { (coffee; stop [] tea; stop)) }
$$

Specification S2 in the example above shows that we had to trade-in some conciseness of specifications for clarity of the semantics. We believe that the semantics of logical disjunction will be better understood by most specifiers than the semantics of non-determinism.

Example 8 In example 4 of the transmission protocol, there was no intended implementation freedom. Since the specification $T P_{\text {spec }}$ does not contain disjuncts, the only possible implementation (modulo bisimulation equivalence) is the specification itself.

The following proposition confirms that the $\bigvee$-operator behaves like logical disjunction.

Proposition 7 Let $s, t \in \mathcal{S}$ be specifications, and $p \in \mathcal{P}$ be a process. Then $p \vDash(s \bigvee t) \Leftrightarrow(p \vDash s) \vee(p \vDash t)$.

$$
\begin{aligned}
& \text { Proof. } \quad p \models(s \bigvee t) \\
& \Leftrightarrow \quad\{\text { definition of } \models \text { \} } \\
& \left.\exists x:(s \bigvee t) \leftrightarrow^{*} x \wedge \text { (conditions } \models_{1} \text { and } \models_{2} \text { hold for }(p, x)\right) \\
& \Leftrightarrow \quad\{\text { lemma } 1.2\} \\
& \exists x:\left(s \leftrightarrow^{*} x \vee t \leftrightarrow^{*} x\right) \wedge(\ldots) \\
& \Leftrightarrow \quad\{\text { distr. of } \wedge \text { over } \vee \text { and distr. of } \exists\} \\
& \left(\exists x: s \leftrightarrow^{*} x \wedge(\ldots)\right) \vee\left(\exists x: t \leftrightarrow^{*} x \wedge(\ldots)\right) \\
& \Leftrightarrow \quad\{\text { definition of } \models \text { \} } \\
& p \vDash s \vee p \models t
\end{aligned}
$$

Because of this connection with logical disjunction, $V$ also enjoys the following properties.

Corollary 8 Let $r, s, t \in \mathcal{S}$ be specifications, and let $p \in \mathcal{P}$ be a process. Then:

1. $p \models s \Leftrightarrow p \models(s \bigvee s)$ (idempotency);

2. $p \models(s \bigvee t) \Leftrightarrow p \models(t \bigvee s)$ (symmetry); 
3. $p \vDash(r \bigvee(s \bigvee t)) \Leftrightarrow p \models((r \bigvee s) \bigvee t)$ (associativity).

It is not hard to see, that the equivalence over processes induced by the specification technique $\langle\mathcal{S}, \models\rangle$ is precisely strong bisimulation equivalence.

Proposition 9 (process equivalence)

Let $p, q \in \mathcal{P}$ be processes, then $p \sim q \Longleftrightarrow \forall s \in \mathcal{S}:(p \vDash s \Leftrightarrow q \vDash s)$.

Proof. (sketch) The proof for this proposition is similar to the proof that bisimulation equivalence is characterised by Hennessy-Milner logic in [Mil89, p.229]. It involves giving alternative characterisations of bisimulation and satisfaction as limits of descending chains of approximating relations. These are then used to prove the proposition by induction.

We can also show that all other operators of the specification language distribute over disjunction. This will be a useful property when we want to establish a normal form for specifications.

Proposition 10 Let $r, s, t \in \mathcal{S}$ be specifications, and let $p \in \mathcal{P}$ be a process. Then the following distributivity properties hold:

1. $p \models((s \bigvee t) \square r) \Leftrightarrow p \models((s[r) \bigvee(t] r))$;

2. $p \vDash((s \bigvee t)|[G]| r) \Leftrightarrow p \vDash((s|[G]| r) \bigvee(t|[G]| r))$;

3. $p \vDash((s \bigvee t) \bigvee r) \Leftrightarrow p \models((s \bigvee r) \bigvee(t \bigvee r))$.

Proof.

1. From left-to-right: Assume $p \models((s \bigvee t)[r)$. Then, by definition 6, there exists an $x$ such that $((s \bigvee t) \square r) \leftrightarrow^{*} x$ and conditions $\left(\models_{1}\right)$ and $\left(\models_{2}\right)$ hold for $p$ and $x$. Inspection of the inference rules for $\bigvee$ and $\square$ results in the following cases:

$x=s^{\prime}\left[r^{\prime}\right.$, where $s \uplus^{*} s^{\prime}$ and $r \uplus^{*} r^{\prime}$ : Since $((s[r) \bigvee(t \square r)) \mapsto(s[r)$ and the fact that $\leftrightarrow \circ \hookrightarrow^{*}=\hookrightarrow^{*}$, we also have $\left(\left(s[r) \bigvee(t[r)) \leftrightarrow^{*} x\right.\right.$, and we are done.

$x=t^{\prime}\left[r^{\prime}\right.$, where $t \leftrightarrow^{*} t^{\prime}$ and $r \leftrightarrow^{*} r^{\prime}$ : Similarly.

From right-to-left: similar.

2. $((s \bigvee t)|[G]| r)$ and $((s|[G]| r) \bigvee(t|[G]| r))$ have isomorphic transition systems. Both specifications have the following $\mapsto$-derivatives: $s|[G]| r$ and $t|[G]| r$. Neither specification has any other derivatives.

3. Follows from the idempotency, symmetry and associativity of $\bigvee$. 


\subsection{Refinement}

The definition of satisfaction above, naturally induces a refinement ordering over specifications. A specification $s$ refines a specification $t$ in case the set of processes satisfying $s$ is a subset of the set of processes satisfying $t$, i.e. $\{p \in \mathcal{P} \mid p \models s\} \subseteq$ $\{p \in \mathcal{P} \mid p \models t\}$. However, generalising definition 6, we can also give an inductive characterisation of refinement:

\section{Definition 11 (Refinement)}

Refinement is the largest relation $\sqsubset \subseteq \mathcal{S} \times \mathcal{S}$ such that, $s \sqsubseteq t$ implies that for each $s^{\prime}$ such that $s \leftrightarrow^{*} s^{\prime}$, there exists a $t^{\prime}$ such that $t \leftrightarrow^{*} t^{\prime}$ and, for each $\mu \in L \cup\{i\}$ the following holds:

(i) Whenever $s^{\prime} \stackrel{\mu}{\longrightarrow} s^{\prime \prime}$ then, for some $t^{\prime \prime}, t^{\prime} \stackrel{\mu}{\longrightarrow} t^{\prime \prime}$ and $s^{\prime \prime} \sqsubseteq t^{\prime \prime}$; and

(ii) Whenever $t^{\prime} \stackrel{\mu}{\longrightarrow} t^{\prime \prime}$ then, for some $s^{\prime \prime}, s^{\prime} \stackrel{\mu}{\longrightarrow} s^{\prime \prime}$ and $s^{\prime \prime} \stackrel{\sim}{\sim} t^{\prime \prime}$.

This definition simply states that $s$ is a refinement of $t$ if there is a disjunct $t^{\prime}$ in $t$ for each disjunct $s^{\prime}$ in $s$, such that $s^{\prime}$ is "bisimilar" to $t^{\prime}$. The following theorem shows that $\sqsubseteq$ is indeed a characterisation of refinement for the specification technique $\langle\mathcal{S}, \models\rangle$.

Theorem 12 Let $s, t \in \mathcal{S}$ be specifications. Then $s \sqsubseteq t \Longleftrightarrow\{p \in \mathcal{P} \mid p \models s\} \subseteq\{p \in \mathcal{P} \mid p \vDash t\}$

Proof. (sketch) The proof for this theorem goes very much along the lines of the proof in [Mil89, p.229] that bisimulation is characterised by Hennessy-Milner logic. It involves giving alternative definitions for $\check{\sim}$ and $\models$ as decreasing $\omega$-sequences of approximating relations. We then use these to prove the given theorem by induction.

Proposition 13 Let $s, t, r \in \mathcal{S}$ be specifications, and let $p \in \mathcal{P}$ be a process. Then the following laws for disjunction will hold:

1. $s$ ᄃ $s \bigvee t$;

2. $t \subseteq i \subsetneq s \bigvee$;

3. If $s \sqsubseteq r$ and $t \sqsubseteq r$, then $s \bigvee t \sqsubseteq r$.

In other words, $s \bigvee t$ is the least upper bound of $s$ and $t$ with respect to the refinement ordering.

Proof. 1. and 2. follow immediately from definition 11, because $s \hookrightarrow^{*} s^{\prime}$ implies $(s \bigvee t) \leftrightarrow^{*} s^{\prime}$ (using lemma 1), and similarly for $t$.

3 . We prove that the assumption that there is a specification $r$, such that $s \underset{\sim}{\sqsubseteq}$ and

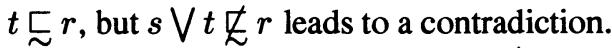

According to definition 11, $s \bigvee t \not Z r$ can only hold, if there exists an $x$, such that $(s \bigvee t) \leftrightarrow^{*} x$, and for all $r^{\prime}$ such that $r \uplus^{*} r^{\prime}$ either of the two conditions of defini- 
tion 11 does not hold. However, if $(s \bigvee t) \leftrightarrow^{*} x$, then (by lemma 1) either $s \leftrightarrow^{*} x$ or $t \leftrightarrow^{*} x$. Since we assumed that $s \sqsubseteq r$ and $t \sqsubset r$, there must exist an $r^{\prime}$ such that $r \leftrightarrow^{*} r^{\prime}$ and $x$ and $r^{\prime}$ satisfy the two conditions, which gives us the contradiction.

Next, we show that refinement, $\sqsubset$, is a (pre-)congruence. That is, refinement is preserved by all specification operators.

Proposition 14 Let $s_{1}, s_{2}, t \in \mathcal{S}$ be specifications, such that $s_{1} \sqsubseteq s_{2}$, then

1. $a ; s_{1} \sqsubseteq a ; s_{2}$

2. $s_{1}\left[t \tilde{t} s_{2}[t\right.$

3. $s_{1}|[G]| t \check{c} s_{2}|[G]| t$

4. $s_{1} \bigvee t \sqsubseteq s_{2} \bigvee t$

Proof. The first case is trivial. The other cases can easily be proved by constructing a relation that contains the pair (LHS,RHS) and then showing that this relation is contained in $\sqsubset$. Here, we prove just the last case.

Consider the relation $\left\{\left(s_{1} \bigvee t, s_{2} \bigvee t\right) \mid s_{1} \sqsubseteq s_{2}\right\} \cup \sqsubseteq$. Whenever $s_{1} \bigvee t \leftrightarrow^{*} x$ then either of the following two cases holds:

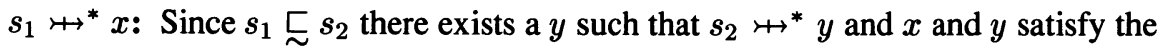
two conditions of definition 11. Since $\left(s_{2} \bigvee t\right) \mapsto s_{2}$, also $\left(s_{2} \bigvee t\right) \leftrightarrow^{*} y$. $t \leftrightarrow^{*} x$ : Since $\left(s_{2} \bigvee t\right) \mapsto t$, also $\left(s_{2} \bigvee t\right) \leftrightarrow^{*} x$, and we are done.

\section{APPLICATIONS}

In [Hoa85], Hoare gives some examples in which the non-deterministic or, $\Pi$, is used for loosely specifying change-giving machines in CSP. These specifications can be expressed equally well in our notation, although their interpretation is slightly different.

Example 9 Consider the following specification of a change-giving machine, which always gives the right change in one of two combinations:

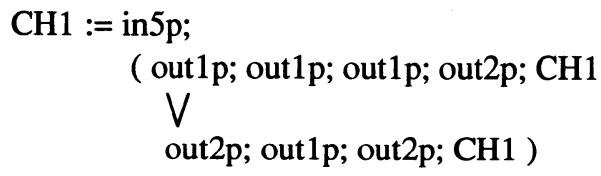

This specification leaves open how the change should be given. Valid implementations are those which always return one of two possible combinations of change, but also those which return different combinations on each invocation. For example, the implementation given by $\mathrm{CH}_{-} \mathrm{I} 1$, which alternates between the two possible combinations, satisfies $\mathrm{CH} 1$. 


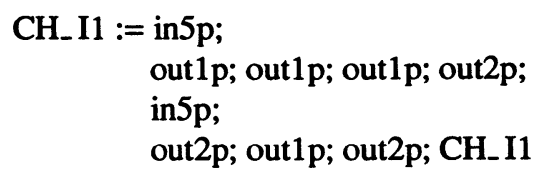

Example 10 We saw that $\mathrm{CH} 1$ allows implementations that give different combinations of change on each invocation. The following specification allows only implementations that always give the same combination, but it leaves open which combination it will be.

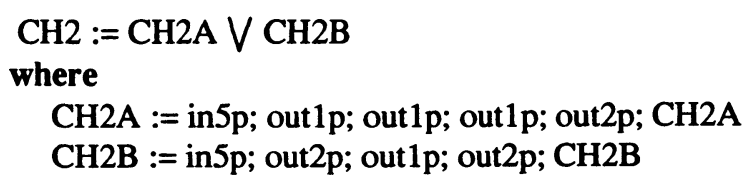

Although CSP's $\Pi$ is intended to play a similar role to logical disjunction, CSP's failures preorder allows also implementations that replace the non-deterministic choice by a deterministic one. This will then give the user a choice, at "run-time", which implementation s/he wants. For example, if the specifications $\mathrm{CH} 1$ and $\mathrm{CH} 2$ had been written with a non-deterministic choice between the alternatives, then both would have allowed the following implementation:

CH_I2 := in5p;

( out1p; out1p; out1p; out2p; $\mathrm{CH}_{-} \mathrm{I} 2$

[]

out2p; out1p; out2p; CH_I2)

which gives the user a chance to influence which combination of change s/he will get. However, the semantics of $\bigvee$ does not allow $\mathrm{CH}_{-} \mathrm{I} 2$ as an implementation of either $\mathrm{CH} 1$ or $\mathrm{CH} 2$, i.e. $\mathrm{CH}_{-} \mathrm{I} 2 \not \models \mathrm{CH} 1, \mathrm{CH} 2$.

\subsection{The most undefined specification}

The disjunction operator can easily be generalised to work over a set of arguments. For $S$ a set of specifications, $\bigvee S$ denotes the disjunction of all the specifications $s \in S$. The semantics is defined by the following family of axioms:

$$
\frac{-}{\bigvee S \mapsto s}(s \in S)
$$

In the same fashion, choice, [, can be generalised to $\Sigma S$, with $\Sigma\{\}=$ stop.

Using these generalised operators, we can define the most undefined specification, i.e. the specification that allows all processes as implementations, provided the alphabet of labels is finite. 


$$
U:=\bigvee\{\Sigma\{\mathbf{a} ; U \mid \mathbf{a} \in \mathbf{A}\} \mid \mathbf{A} \subseteq \mathcal{L}\}
$$

Example 11 Let $\mathcal{L}=\{a, b\}$ be the alphabet. Then the most undefined specification $U$ is given by:

$$
U:=\operatorname{stop} \bigvee \mathbf{a} ; U \bigvee \mathbf{b} ; U \bigvee(\mathbf{a} ; U[] \mathrm{b} ; U)
$$

This most undefined specification is very useful for partial specification. Whenever we want to leave open the behaviour at a certain point, we can just plug-in $U$. Later on, this can be refined to anything, thus achieving complete implementation freedom.

\section{CONCLUSION}

Many others before us have recognised the limited expressiveness of process algebras for the specification of non-deterministic, concurrent processes. A common approach has been to define a logic, separate from the process description language, for the specification of properties of processes (e.g. Hennessy-Milner Logic (HML) [HM85] and modal $\mu$-calculus [Koz83]). A clear drawback is that specifications and implementations are in different notations. Step-wise refinement is not possible, and verification can only be done a posteriori. In order to alleviate this problem, there have been some attempts to introduce the process structuring operators into these logics. In [Hol89], HML is extended with the CCS operators, and in [BGS89], the same is done for a fragment of the $\mu$-calculus. Unfortunately, these languages have a denotational semantics: each specification is associated with the set of processes that satisfy it. Verifying whether a process satisfies a specification amounts to checking whether it is in that set. Alternatively, the correctness of an implementation can be verified through (in-)equational reasoning.

Another way to increase the expressive power of process algebraic specifications is introduced in [Lar90b], where transitions are decorated with modalities. A distinction is made between required and allowed transitions. Bisimulation equivalence is then generalised to a refinement relation that ensures that the more concrete specification requires more and allows less. It is also possible to define the equivalent of logical conjunction operationally in this model [LSW95]. In fact, it has been shown that the specification technique thus obtained is as expressive as a restricted version of HML [BL92]. The restriction is caused by the inability to adequately express disjunction. However, modal transition systems can be extended with disjunction in the same way we have extended labelled transition systems with disjunction in this paper. Would this then create a specification technique with the full power of HML?

\section{ACKNOWLEDGEMENTS}

We would like to thank Rom Langerak for discussing ideas that led to this paper, and the anonymous referees for their useful comments. 


\section{REFERENCES}

[BGS89] A. Bouajjani, S. Graf, and J. Sifakis. A logic for the description of behaviours and properties of concurrent systems. LNCS 354, pages 398410, 1989.

[BL92] G. Boudol and K.G. Larsen. Graphical versus logical specifications. Theoretical Computer Science, 106:3-20, 1992.

[BSS87] E. Brinksma, G. Scollo, and C. Steenbergen. LOTOS specifications, their implementations and their tests. In PSTV VI, pages 349-360, 1987.

[HM85] M. Hennessy and R. Milner. Algebraic laws for nondeterminism and concurrency. Journal of the ACM, 32(1):137-161, January 1985.

[Hoa85] C.A.R. Hoare. Communicating Sequential Processes. Prentice-Hall, 1985.

[Hol89] S. Holmström. A refinement calculus for specifications in HennessyMilner logic with recursion. Formal Aspects of Computing, 1(3):242272, 1989.

[Koz83] D. Kozen. Results on the propositional $\mu$-calculus. Theoretical Computer Science, 27(3):333-354, December 1983.

[Lar90a] K.G. Larsen. Ideal specification formalism = expressivity + compositionality + decidability + testability $+\cdots$. In CONCUR'90, LNCS 458, pages 33-56, 1990.

[Lar90b] K.G. Larsen. Modal specifications. In J. Sifakis, editor, Automatic Verification Methods for Finite State Systems: Proceedings, LNCS 407, pages 232-246. Springer-Verlag, 1990.

[Led91] G. Leduc. On the Role of Implementation Relations in the Design of Distributed Systems using LOTOS. PhD thesis, University of Liège, Belgium, June 1991.

[Led92] G. Leduc. A framework based on implementation relations for implementing LOTOS specifications. Computer Networks and ISDN Systems, 25:23-41, 1992.

[LSW95] K.G. Larsen, B. Steffen, and C. Weise. A constraint oriented proof methodology based on modal transition systems. In E. Brinksma, editor, TACAS'95, LNCS 1019, pages 17-40, 1995.

[Mil89] R. Milner. Communication and Concurrency. Prentice-Hall, 1989.

[NH84] R. de Nicola and M.C.B. Hennessy. Testing equivalences for processes. Theoretical Computer Science, 34:83-133, 1984.

Ir M.W.A. Steen obtained an MSc(Eng) in Computer Science from the University of Twente, The Netherlands, in 1993. He is currently completing a PhD degree in Computer Science at the University of Kent at Canterbury. His current research focuses on partial specification in process algebra, in particular on techniques for consistency checking and composition. Furthermore he has worked on application of these, and other, formal techniques in the area of Open Distributed Processing.

Dr. H. Bowman, Dr. J. Derrick and Dr.Ir. E.A. Boiten are lecturers in the Computing Laboratory at the University of Kent. 